\title{
The interplay of received social support and self-regulatory factors in smoking cessation
}

\author{
Sibylle Ochsner ${ }^{\text {* }}$, Aleksandra Luszczynska ${ }^{\text {b,c }}$, Gertraud Stadler ${ }^{\mathrm{d}}$, Nina Knolle, \\ Rainer Hornung ${ }^{a}$ and Urte Scholz ${ }^{f}$ \\ ${ }^{a}$ Department of Psychology, Social and Health Psychology, University of Zurich, Zurich, \\ Switzerland; ${ }^{b}$ Department of Psychology, University of Social Sciences and Humanities, Wroclaw, \\ Poland; 'Trauma, Health, \& Hazards Center, University of Colorado, Colorado Springs, CO, \\ USA; 'Department of Sociomedical Sciences, Mailman School of Public Health, Columbia \\ University, New York, NY, USA; 'Department of Educational Science and Psychology, Division \\ Health Psychology, Freie Universität Berlin, Berlin, Germany; ${ }^{\prime}$ Department of Psychology, \\ Developmental and Health Psychology, University of Konstanz, Konstanz, Germany
}

\begin{abstract}
Objective: In smoking cessation, individual self-regulation and social support have both proven to be useful. However, the roles of self-regulatory processes and social support are mostly examined separately. The present study aims at examining the unique and joint interactive effects of self-regulation as specified in the health action process approach (HAPA) and social support on smoking cessation. The study tested whether social support can compensate for low levels of self-regulation or whether synergistic effects emerge.

Design \& Measures: Around a self-set quit date, 99 smokers completed baseline questionnaires on HAPA-variables, smoking-specific received social support and smoking cessation (continuous abstinence and point prevalence), with a follow-up conducted approximately 29 days after the quitdate.

Results: Social support moderated the association between volitional selfefficacy and smoking, as well as coping planning and smoking but not between action planning and smoking. No compensatory effect of social support for lower levels of individual regulation emerged but the combination of high levels of the individual variables and social support was related to successful smoking cessation, indicating a synergistic effect.

Conclusions: The results confirm the importance of examining both selfregulation and social factors in smoking cessation. This should be considered when developing future interventions for smoking cessation.
\end{abstract}

Keywords: health behaviour change; smoking cessation; received social support; health action process approach

Smoking causes a higher risk for many diseases such as cancer, stroke or cardiovascular disease. Thereby, smoking behaviour is one significant predictor of the leading causes of death (Mokdad, Marks, Stroup, \& Geberding, 2004). Smoking is a multifactorial addiction including physical, mental and social factors. Previous research confirmed individual self-regulation and social factors (e.g. social support and social control) to be

*Corresponding author. Email: sibylle.ochsner@psychologie.uzh.ch 
important in the initiation of quit attempts and staying smoke-free (e.g. Gulliver, Hughes, Solomon, \& Dey, 1995; Gwaltney, Metrik, Kahler, \& Shiffman, 2009; Park, Tudiver, Schultz, \& Campbell, 2004). Nonetheless, smoking cessation and general health behaviour change studies rarely look at the unique and joint influences of individual and social factors, which was the aim of the current paper.

\section{Health action process approach (HAPA)}

The theoretical background model of the present study was the HAPA (Schwarzer, 2008). The HAPA model distinguishes between a motivational phase and a volitional phase during the process of behaviour change. The motivational phase results in the formation of an intention to change the behaviour when people have high risk awareness, hold more positive than negative outcome expectancies and have high motivational self-efficacy. To translate intentions into behaviour in the volitional phase, volitional self-efficacy, action planning and coping planning are specified as post-intentional predictors of the behaviour. Volitional self-efficacy refers to optimistic beliefs about one's capability to maintain behaviour change over a longer period of time and deal with obstacles that arise as well as optimistic beliefs about not returning to smoking after a lapse or relapse has occurred (Schwarzer \& Luszczynska, 2008). Action planning is defined as forming specific plans about when, where and how to perform a certain behaviour. Coping planning refers to the imagination of scenarios that hinder one from performing the intended behaviour and to developing plans to cope with such difficult situations. The HAPA model has been applied successfully in the context of smoking (e.g. Schwarzer \& Luszczynska, 2008; Scholz, Nagy, Gohner, Luszczynska, \& Kliegel, 2009). Moreover, it has been demonstrated to be of good applicability, universality and predictive validity regarding different health behaviours and different populations (Schwarzer, 2008).

\section{Social support}

Social support is an interactive process between a provider and a receiver, and it refers to the function and quality of social relationships. It can be differentiated into perceived and received social support (e.g. Schwarzer \& Knoll, 2010). Perceived social support comprises the anticipated available support from the social network if it is needed. This general expectation of support in the future somewhat resembles a personality disposition related to optimism and is relatively stable (Sarason, Sarason, \& Shearin, 1986). Received social support refers to retrospective reports of actual support transactions in the past (Schwarzer \& Knoll, 2010). According to the different definitions and measurements, perceived and received social support do not necessarily need to have much in common (Sarason et al., 1986). In the context of smoking cessation, studies investigated perceived and received social support (e.g. Carlson, Goodey, Bennett, Taenzer, \& Koopmans, 2002; Gulliver et al., 1995). Most studies, however, have focused on perceived social support. The current study examined received social support from the non-smoking partner in line with the fact that the partner is frequently the most important source of social support (Schwarzer \& Gutierrez-Dona, 2005). 


\section{Combination of individual and social factors}

As outlined above, there are only few studies that try to combine individual and social factors in health behaviour change. There is positive evidence for the main effects of social support and individual regulation from the information-motivation-behavioural skills model (for an overview, see Fisher, Fisher, Amico, \& Harman, 2006). Moreover, a study from Scholz, Ochsner, Hornung, and Knoll (2013) demonstrated the first evidence for the beneficial effects from received social support over and above selfregulation constructs of the HAPA model. Research within the frameworks of the theory of planned behaviour (Ajzen, 1991), social cognitive theory (Bandura, 2001) and the transtheoretical model of behaviour change (Prochaska \& DiClemente, 1983) that examined self-regulation and added social support to predict intentions or behaviour showed mixed results (e.g. Andersen, 2006; Anderson, Winett, \& Wojcik, 2007; Hamilton \& White, 2008).

Social support has also been examined as a moderator of the link between selfregulation and health behaviours. Povey, Conner, Sparks, Rhiannon, and Shepherd (2000) added perceived social support to the theory of planned behaviour in the context of healthy eating. They found no main effect of perceived social support on intentions, but social support was found to act as a moderator of the association between perceived behavioural control and intention and of the association between attitude and intention (Povey et al., 2000). In the latter, perceived social support was positively related to intentions at all levels of attitudes. However, as levels of social support increased, attitude became a stronger predictor of intentions showing that as social support increased, the power of attitude to predict intentions increased as well. This interaction effect indicates a synergistic effect of individual regulation and social support. A different picture emerged for the moderating effect of perceived social support on the association between perceived behavioural control and intentions. At high levels of social support, perceived behavioural control did not predict intention, whereas at lower levels of social support, perceived behavioural control was a strong positive predictor of intentions, thereby indicating a compensatory effect of social support. Effects on behaviour were not tested.

Warner et al. (2011) found an interaction effect of self-efficacy and received social support on perceived autonomy in multimorbid individuals. In individuals with lower self-efficacy, social support was positively related to the perception of autonomy, showing that they compensated their low levels of self-efficacy with received social support. For individuals with higher self-efficacy, higher levels of social support were interfering with autonomy; the combination of high levels of social and individual resources interfered with perceived autonomy in this sample (Warner et al., 2011). It remains unclear, however, whether these findings can be transferred to health behaviour change.

Overall, there is a considerable lack of research with regard to interacting effects of social support and, in particular, received social support and individual regulation. This is surprising in that people usually do not change their (health) behaviours in isolation, but within certain social contexts (e.g. at home, at work). Results from the few studies available suggest two possible outcomes of an interaction between social and individual variables: compensating or synergistic effects. The compensating function of received social support for deficits in individual self-regulation can be hypothesised for the following reasons: (1) partners potentially know their smoking spouses' weaknesses in 
individual self-regulation best (e.g. Sillars \& Scott, 1983); thus, support could compensate for self-regulation deficits. (2) For most smokers, trying to quit smoking is a stressful situation (McMahon \& Jason, 1998) that may be even worse for individuals with lower individual resources. In this taxing situation, social support could be buffering and be most helpful for those individuals who need it the most because of their low levels of self-regulation (see also Warner et al., 2011 for compensating function). Alternatively, the synergistic effects of social support and individual regulation variables as for example found by Povey et al. (2000) for the association of attitudes and intentions may also appear in the context of smoking cessation. Quitting smoking is a very difficult endeavour with very high rates of relapse (e.g. relapse rates of over $70 \%$ after one month, Hughes, Keely, \& Naud, 2003). Thus, self-regulation alone might not suffice but smokers might be in need of both high self-regulation skills and high levels of received social support from their spouses.

\section{Aim of the study}

To the best of our knowledge, no studies on the joint effects of received social support and individual regulation with regard to smoking cessation exist so far. Therefore, the aim of the current study was to examine whether smoking-specific received social support and individual self-regulation, in particular volitional self-efficacy, action planning and coping planning interact with regard to smoking cessation. Due to the lack of research on the joint effects of social and individual variables in the context of health behaviour, in general, compensating or synergistic effects of social support and individual regulation were tested as two alternative hypotheses.

\section{Method}

\section{Sample and procedure}

The sample consisted of 106 smoking participants at T1. Of these, $72.6 \%(n=77)$ were men and the mean age was 40.67 years ( $\mathrm{SD}=10.03$, ranging from 19 to 72 ). The majority of participants were married $(n=69,65.1 \%), 34.9 \%(n=37)$ were not married but according to inclusion criteria, all participants were in a committed relationship and cohabiting with a non-smoking partner of the opposite sex. In large parts, participants had children $(n=62,58.5 \%)$. Most participants were currently employed $(n=86$, $81.1 \%)$ and reported having attended nine years of schooling $(n=75,70.8 \%)$. Overall, participants had a strong intention to quit smoking (see Table 1).

The study was part of the larger project 'dyadic and individual regulation to end chronic tobacco use (DIRECT)' funded by the Swiss National Science Foundation (100014_124516). The sample was recruited via newspapers, web pages and a marketing research institution. The inclusion criterion was smoking at least one cigarette per day (in line with the definition of daily smokers by the World Health Organization (WHO, 1998). In this study, 99\% of the participants smoked more than one cigarette daily). Moreover, being in a committed relationship or married to a non-smoking partner for at least one year, cohabiting with the partner for at least six months and wanting to quit smoking were further inclusion criteria. The exclusion criteria were: participation in a professional programme on smoking cessation, being pregnant or partner being pregnant, working in shift work and insufficient comprehension of the German 
Table 1. Means, standard deviations, ranges, internal consistency and correlations of main study variables and potential covariates.

\begin{tabular}{|c|c|c|c|c|c|c|c|c|c|c|c|c|c|c|c|}
\hline & $M$ & SD & Range & $\alpha$ & Point $\mathrm{P}$ & Vol SE & $\mathrm{AP}$ & $\mathrm{CP}$ & Intent & Support & T1 to quit & Quit to $\mathrm{T} 2$ & Soc D & Age & Sex \\
\hline Cont $\mathrm{Ab}$ & & & $1-2$ & & $44^{* * * *}$ & .01 & -.10 & -.05 & $-.22^{*}$ & .05 & -10 & .12 & .01 & -.14 & -.06 \\
\hline Point $\mathrm{P}$ & & & $1-2$ & & & .02 & -.16 & -.03 & $-.19^{\#}$ & .10 & .01 & .04 & -.03 & -.02 & -.02 \\
\hline Vol SE & 3.32 & .97 & $1-5$ & .82 & & & .07 & .13 & .11 & -.03 & $-.28^{* *}$ & .08 & .07 & $-.22^{*}$ & -.06 \\
\hline AP & 4.12 & 1.06 & $1-6$ & .78 & & & & $.74^{* * * *}$ & $.27^{* *}$ & $.25^{* * *}$ & -.13 & .02 & .05 & -.05 & -.13 \\
\hline $\mathrm{CP}$ & 3.32 & 1.00 & $1-5.75$ & .84 & & & & & $.19^{*}$ & $.30^{* * *}$ & -.16 & .06 & .12 & -.10 & -.06 \\
\hline Intent & 5.53 & .66 & $4-6$ & & & & & & & .07 & $-.32^{* * *}$ & .08 & -.17 & .01 & .03 \\
\hline Support & 3.20 & .86 & $1-5.14$ & .77 & & & & & & & $-.19^{\#}$ & .09 & $.19^{\#}$ & -.14 & -.09 \\
\hline T1 to quit & 17.14 & 6.86 & $8-39$ & & & & & & & & & -.13 & .15 & .14 & .05 \\
\hline Quit to $\mathrm{T} 2$ & 29.17 & 7.82 & $21-58$ & & & & & & & & & & .09 & -.13 & .04 \\
\hline Soc D & 1.69 & .17 & $1.25-2$ & .61 & & & & & & & & & & .09 & $.20^{*}$ \\
\hline Age & 40.67 & 10.03 & $19-72$ & & & & & & & & & & & & .07 \\
\hline
\end{tabular}

Notes: Cont Ab: continuous abstinence, Point P: point prevalence, Vol SE: volitional self-efficacy, AP: action planning, CP: coping planning, intent: behavioural intentions, support: received social support, T1 to quit: duration between T1 and quit date (days), quit to T2: duration between quit date and T2 (days), Soc D: social desirability, Sex: $1=$ men, 2 = women. $N=106 .{ }^{\#} p<.10,{ }^{*} p<.05,{ }^{* *} p<.01,{ }^{* * *} p<.001$. 
language. Non-smoking partners also participated in the DIRECT project, but were not focused on in this study.

Prior to the baseline assessment, the participants were emailed a questionnaire (T0) which was completed online. The baseline assessment (T1) then took place at the university laboratory. ${ }^{1}$ After receiving information about the study and the procedure, the participants signed an informed consent form and received a personal code to ensure anonymity. Then they announced their self-set quit date, which was on average 17 days (range 8-39 days) after T1, and completed a questionnaire. Moreover, smoking was biochemically verified with a carbon monoxide test of expired air. On average, 29 days (range 21-58 days) after the quit date, the respondents returned to the lab for the second assessment (T2). The participants again completed a questionnaire and biochemical verification of smoking status. After finishing T2, they received a compensation of 100 Swiss Francs. Six months after the quit date, the last assessment (T3) took place at the lab and the respondents received again 100 Swiss Francs. Additionally, there was a diary phase with 32 diary days between $\mathrm{T} 1$ and $\mathrm{T} 2$ around the self-set quit date. In this study, the data from baseline and $\mathrm{T} 2$ are focused on, whereas the diary data and six-month follow-up were not included. The rationale for examining potential joint effects of smoking-specific received social support and individual self-regulation before the quit date and post-quit date smoking cessation in the short-term rather than a longer-term (6 months) perspective lies in the chosen constructs. In contrast to perceived social support, received social support is not assumed to be stable over time, but a measure of retrospective support transactions from the partner. It is thus highly likely that either success or failure in smoking cessation during the six-month period will impact social support received from the partner. Likely, the partner will adapt their support provision to the outcome over time. Thus, including received social support before the quit date to predict smoking cessation six months later may not be justified. In contrast, received support and joint effects of received support with volitional constructs are rather likely to display short-term effects on smoking cessation after the quit date (i.e. as assessed at the one-month follow-up at T2). The diary data address different research questions and are thus also not focused on in the present study. All the participants were treated in accordance to the ethical guidelines of the Helsinki Declaration 2000.

\section{Measures}

At Time 0 , in the initial questionnaire, sociodemographic variables were assessed. At Time 1, all psychosocial constructs and at Time 2, smoking cessation was measured. Unless otherwise stated, the response format was a six-point Likert scale ranging from $1=$ completely disagree to $6=$ completely agree. Table 1 presents means, standard deviations and scale reliabilities.

Volitional self-efficacy was measured by four items adapted from Scholz et al. (2009), for example, 'I am confident that after a lapse I can quit smoking for good even if I ... rescheduled my plans several times'.

Action Planning was assessed by five items (Scholz et al., 2009), for example, 'I have made a detailed plan regarding ... when not to smoke'.

Coping Planning was measured by four items (adapted from Sniehotta, Schwarzer, Scholz, \& Schuez, 2005), for example, 'I have made a detailed plan regarding ... what to do in difficult situations in order to act according to my intentions'. 
Behavioural intentions were assessed by a single item (Scholz et al., 2009): 'I intend to quit smoking'.

Smoking-specific received social support was measured by nine items (Burkert, Knoll, \& Scholz, 2005). The participants were asked to think of their partner and how he or she reacted with emotional or instrumental support to the participant in the past seven days, for example, 'My partner ... reminded me of strategies, which help me to resist smoking' or '... comforted me when I was feeling bad because I could not smoke'.

Smoking cessation was assessed in two different ways: (1) with a measure of continuous abstinence and (2) with biochemical verification of point prevalence. Continuous abstinence was measured by a single self-report item: 'Have you smoked since self-set quit date?' Measures of continuous abstinence are the most rigorous and conservative measures of smoking cessation and are therefore often considered as the gold standard. One argument against this strict measure is that it excludes individuals who will achieve life-long abstinence, but who smoke up to a few cigarettes in the first days after the cessation date (Hughes, Keely, Niaura et al., 2003). Therefore, participants are often classified as abstinent if they have smoked a maximum of five cigarettes from the start of the abstinence period (West, Hajek, Stead, \& Stapleton, 2005). Considering this argument, the response format in the present study was $1=$ No or smoking of maximum 5 cigarettes vs. 2 =yes, more than 5 cigarettes. Moreover, as a second measure of the smoking cessation, the point prevalence of smoking at T2 was biochemically verified with a carbon monoxide test of expired air (West et al., 2005). For this test, the Smokerlyzer (Bedfont Instruments, Harrietsham, UK) was used. This method was chosen as it is non-invasive, valid and not biased by the use of nicotine replacement products, as, for example, the assessment with salivary cotinine samples is. The usual cut-off point is $>9$ ppm for indicating smoking (West et al., 2005). In the present study, we therefore categorized participants as $1=$ non smokers $(\leqslant 9 \mathrm{ppm})$ vs. $2=$ smokers $(>9 \mathrm{ppm})$. As carbon monoxide tests can only detect smoking within the previous approximately $24 \mathrm{~h}$ and are thus measures of point prevalence (i.e. they assess smoking only at a single point in time), they are much less strict than measures of continuous abstinence that assess smoking abstinence over a longer time span.

Duration between $T 1$ and quit date, and duration between quit date and $T 2$ were assessed as potential covariates. As the cessation date was self-set, the time between quit date and $\mathrm{T} 1$ or $\mathrm{T} 2$ varied and was therefore measured in days.

Social desirability, age and sex were also assessed as potential covariates, as in Switzerland, where the study was conducted, men and younger individuals smoke more than women and older individuals (Keller, Radtke, Krebs, \& Hornung, 2011). Age was measured in years and sex was coded as $1=$ men and $2=$ women. In order to control for potential bias of self-reported smoking cessation, social desirability was assessed by 16 items from the social desirability scale (SDS-17; Stöber \& Luther, 2001). The response format was dichotomous with $1=$ do not agree vs. 2 =agree, with higher values indicating higher social desirability.

\section{Data analyses}

At $\mathrm{T} 1$, no variable had more than $2 \%$ missing values. At $\mathrm{T} 2$, the variables assessing smoking cessation showed $7.5 \%$ missing values. Numbers of cigarettes smoked at $\mathrm{T} 1$, 
however, were not related to any known mechanism. As there was no significant difference in smoking behaviour at $\mathrm{T} 1$ for dropouts and continuers, and overall the amount of missing data was very small, listwise deletion of missing cases was applied (Graham, 2009). Outliers were treated in accordance with Tabachnick and Fidell (2001). All analyses were conducted with SPSS 20. Main analyses were logistic regression models. The model contained as predictors the covariate, the self-regulation variables volitional self-efficacy, action planning, coping planning, behavioural intentions and received social support. To test for moderation, the interactions of support with the three self-regulation variables were entered. The variables were mean-centred to avoid problems with multicollinearity (Cohen, Cohen, West, \& Aiken, 2003). In reporting the moderation models, we also include findings on the $10 \%$ significance level. The rationale lies in the sample size, as samples with about 100 participants have limited power for detecting moderation effects (see e.g. McClelland \& Judd, 1993). The Johnson-Neyman technique was applied to test the regions of significance of the interaction effects. This technique provides the range of the moderator within which the simple slope of the dependent variable on the predictor is significantly different from zero (Preacher, Curran, \& Bauer, 2006).

\section{Results}

\section{Descriptive statistics}

At $\mathrm{T} 1$, the average number of daily smoked cigarettes was 16.59 ( $\mathrm{SD}=8.52$, range $1-40)$ and at $\mathrm{T} 2$ on average $5.27(\mathrm{SD}=6.97$, range $0-40)$, showing a significant reduction from $\mathrm{T} 1$ to $\mathrm{T} 2(F(1,97)=195.67, p=.001)$. According to the measure of continuous abstinence, 34 (32.1\%) participants did not smoke between their quit date and $\mathrm{T} 2$. The less strict measure of point prevalence verified by expired carbon monoxide resulted in $67(63.2 \%)$ non-smokers at $\mathrm{T} 2$. The 34 participants reporting continuous abstinence were part of the 67 participants identified as non-smokers by point prevalence. The discrepancy between these two measures of smoking cessation can be explained by the participants who reported smoking between their quit date and $\mathrm{T} 2$ but were smoke-free at $\mathrm{T} 2$ and also by the fact that the biochemically verifiable time window for carbon monoxide is about one day, indicating that participants who did not smoke the day before T2 were counted as smoke-free with this measure. There is no practicable way to objectively verify abstinence over a longer time span, but the point prevalence measure provides at least a minimum assurance concerning abstinence at the follow-up point (Hughes, Keely, Niaura et al., 2003; West et al., 2005).

Of the initial 106 participants, $99(93.4 \%)$ completed the T2 follow-up. No significant differences emerged between dropouts and participants who completed both questionnaires regarding action planning, coping planning, smoking cessation, social desirability, sex, marital status, having children and education. There were significant differences between dropouts and continuers for received social support, $F(1,103)$ $=9.90, p=.002(M=2.25, \mathrm{SD}=1.08$ for dropouts, $M=3.27, \mathrm{SD}=.81$ for continuers), volitional self-efficacy, $F(1,104)=4.22, p=.043(M=2.54, \mathrm{SD}=.86$ for dropouts, $M=3.37, \mathrm{SD}=.96$ for continuers $)$ and age, $F(1,105)=4.12, p=.045 \quad(M=48.00$, $\mathrm{SD}=16.15$ for dropouts, $M=40.15, \mathrm{SD}=9.37$ for continuers). Retired participants were more likely to dropout, whereas employed participants were more likely to continue 
$\left(\chi^{2}(4)=14.98, p=.005\right)$. However, at $\mathrm{T} 1$, the number of missing values in all these variables did not exceed $2 \%$.

Correlations of volitional self-efficacy, action and coping planning, behavioural intentions, smoking-specific received social support, measures of smoking cessation (continuous abstinence and point prevalence at $\mathrm{T} 2$ ) and the potential covariates duration between $\mathrm{T} 1$ and quit date, duration between quit date and $\mathrm{T} 2$, social desirability, age and sex are displayed in Table 1 . The measures of smoking cessation were positively correlated. Behavioural intentions were correlated negatively with the measures of smoking cessation and positively with action planning and coping planning. The latter two were correlated positively with received social support and were also interrelated. From the potential covariates, sex and duration between quit date and $\mathrm{T} 2$ were not correlated with any predictor or outcome variables. Age was only correlated with volitional self-efficacy and social desirability only with social support at the $10 \%$ level. The duration between $\mathrm{T} 1$ and quit date was associated negatively with volitional self-efficacy, behavioural intentions and social support. As the variability of this time span might have had an effect on the results, the duration between $\mathrm{T} 1$ and quit date was included as covariate in the regression analyses.

\section{Main effects of self-regulation and social support on smoking cessation}

Behavioural intentions emerged as predictor of continuous abstinence (see Table 2) indicating that individuals with higher levels of intentions were more likely to stay abstinent. For point prevalence, however, intentions were not predictive (see Table 3). No main effects of volitional self-efficacy, action planning, coping planning or smoking-specific received social support were observed for either of the smoking cessation measures. There was a tendency that longer duration between baseline assessment and

Table 2. Prediction of continuous abstinence by volitional self-efficacy, action planning, coping planning and behavioural intentions moderated by received social support.

\begin{tabular}{|c|c|c|c|c|c|c|c|c|c|}
\hline \multirow[b]{2}{*}{ Predictor } & \multicolumn{9}{|c|}{ Continuous abstinence (from quit date until T2) } \\
\hline & $b$ & $\begin{array}{l}\text { SE } \\
b\end{array}$ & $\begin{array}{l}\text { Odds } \\
\text { ratio }\end{array}$ & $b$ & $\begin{array}{c}\mathrm{SE} \\
b\end{array}$ & $\begin{array}{l}\text { Odds } \\
\text { ratio }\end{array}$ & $b$ & $\begin{array}{c}\text { SE } \\
b\end{array}$ & $\begin{array}{l}\text { Odds } \\
\text { ratio }\end{array}$ \\
\hline Constant & .68 & .24 & $1.98^{* * *}$ & .80 & .25 & $2.22^{* * * *}$ & .90 & .26 & $2.45^{* * * *}$ \\
\hline Duration $\mathrm{T} 1$ to quit & -.05 & .04 & .95 & -.07 & .04 & $.94^{\#}$ & -.07 & .04 & $.93^{\#}$ \\
\hline Volitional self-efficacy & .08 & .26 & 1.08 & .04 & .25 & 1.05 & .09 & .26 & 1.09 \\
\hline Action planning & -.13 & .33 & .88 & -.22 & .34 & .81 & -.27 & .34 & .77 \\
\hline Coping planning & .02 & .34 & 1.02 & .01 & .34 & 1.01 & -.01 & .35 & 1.00 \\
\hline Behavioural intentions & -.95 & .46 & $.39^{*}$ & -1.02 & .46 & $.36^{*}$ & -.96 & .48 & $.38^{*}$ \\
\hline Received social support & .36 & .34 & 1.43 & .14 & .31 & 1.15 & .21 & .34 & 1.24 \\
\hline $\begin{array}{l}\text { Volitional self-efficacy } \\
\times \text { support }\end{array}$ & -.66 & .37 & $.52^{\#}$ & & & & & & \\
\hline Action planning $\times$ support & & & & -.39 & .27 & .68 & & & \\
\hline Coping planning $\times$ support & & & & & & & -.72 & .30 & $.49^{*}$ \\
\hline
\end{tabular}

Notes: Support: received social support. $N=97 .{ }^{*} p<.10,{ }^{*} p<.05,{ }^{* *} p<.01,{ }^{* * * *} p<.001$. Model volitional self-efficacy $\times$ support: $R^{2}=.12$ (Cox \& Snell), .17 (Nagelkerke); model action planning $\times$ support: $R^{2}=.11$ (Cox \& Snell), .15 (Nagelkerke); model coping planning $\times$ support: $R^{2}=.16$ (Cox \& Snell), 21 (Nagelkerke). 
Table 3. Prediction of point prevalence by volitional self-efficacy, action planning, coping planning and behavioural intentions moderated by received social support.

\begin{tabular}{|c|c|c|c|c|c|c|c|c|c|}
\hline \multirow[b]{2}{*}{ Predictor } & \multicolumn{9}{|c|}{ Point prevalence (T2) } \\
\hline & $b$ & $\begin{array}{c}\mathrm{SE} \\
b\end{array}$ & $\begin{array}{l}\text { Odds } \\
\text { ratio }\end{array}$ & $b$ & $\begin{array}{c}\text { SE } \\
b\end{array}$ & $\begin{array}{l}\text { Odds } \\
\text { ratio }\end{array}$ & $b$ & $\begin{array}{c}\mathrm{SE} \\
b\end{array}$ & $\begin{array}{l}\text { Odds } \\
\text { ratio }\end{array}$ \\
\hline Constant & -.91 & .25 & $.40^{* * * *}$ & -.75 & .24 & $.47^{* * *}$ & -.73 & .24 & $.48^{* *}$ \\
\hline Duration TI to quit & .02 & .04 & 1.02 & .01 & .04 & 1.01 & .01 & .04 & 1.01 \\
\hline Volitional self-efficacy & .12 & .28 & 1.13 & .01 & .26 & 1.01 & .03 & .26 & 1.03 \\
\hline Action planning & -.54 & .37 & .58 & -.56 & .36 & .57 & -60 & .36 & .55 \\
\hline Coping planning & .49 & .37 & 1.64 & .43 & .35 & 1.54 & .48 & .37 & 1.62 \\
\hline Behavioural intentions & -.44 & .39 & .64 & -.45 & .38 & .64 & -.43 & .38 & .65 \\
\hline Received social support & .55 & .36 & 1.74 & .23 & .31 & 1.26 & .28 & .31 & 1.32 \\
\hline $\begin{array}{l}\text { Volitional } \\
\text { self-efficacy } \times \text { support }\end{array}$ & -.80 & .38 & $.45^{*}$ & & & & & & \\
\hline Action planning $\times$ support & & & & -.34 & .29 & .71 & & & \\
\hline Coping planning $\times$ support & & & & & & & -.49 & .31 & .62 \\
\hline
\end{tabular}

Notes: Support: received social support. $N=97 .{ }^{*} p<.10,{ }^{*} p<.05,{ }^{* *} p<.01,{ }^{* * *} p<.001$. Model volitional self-efficacy $\times$ support: $R^{2}=.13$ (Cox \& Snel1), .18 (Nagelkerke); model action planning $\times$ support: $R^{2}=.09$ (Cox \& Snell), .13 (Nagelkerke); model coping planning $\times$ support: $R^{2}=.11$ (Cox \& Snell), 15 (Nage1kerke).

quit date predicted higher likelihood of continuous abstinence $(p<.10)$ in the models for action and coping planning.

\section{Testing the joint effects of received social support and volitional self-efficacy}

To test whether received social support serves a compensating function for low volitional self-efficacy with regard to smoking cessation or whether a synergistic effect emerges, two moderator analyses with the two different measures of smoking cessation were conducted. The first moderator analysis predicted continuous abstinence from the quit date until T2 (see Table 2, first column). The interaction between volitional self-efficacy and smoking-specific received social support was significant at the $10 \%$ level (see Figure 1, left panel). It was further investigated by testing the region of significance, applying the Johnson Neyman technique, resulting in a range of -1.50 to 1.87. These results indicate that for social support values lower than -1.50 and higher
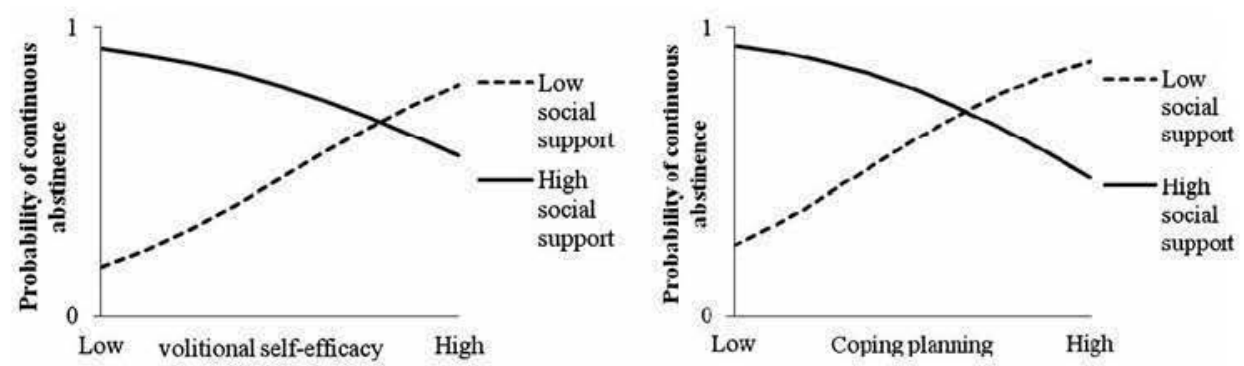

Figure 1. Interaction of volitional self-efficacy/coping planning and received social support on continuous abstinence $(0=$ abstinence, $I=$ smoking). Low social support shown for the empirical minimum (1), high social support for the empirical maximum (5.14). 


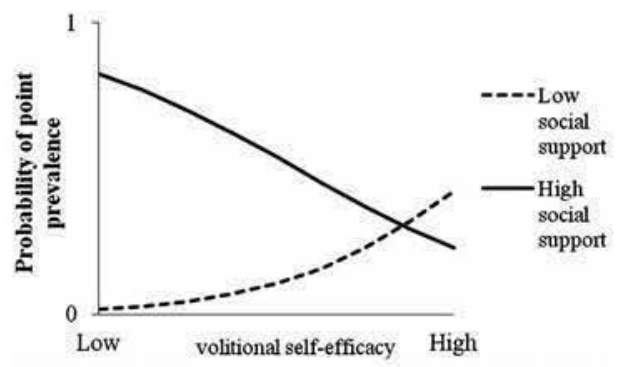

Figure 2. Interaction of volitional self-efficacy and received social support on point prevalence $(0=$ abstinence, $I$ = smoking). Low social support shown for the empirical minimum (1), high social support for the empirical maximum (5.14).

than 1.87 , the association between volitional self-efficacy and continuous abstinence was significant $(p<10)$. As the minimum and maximum of the mean-centred smokingspecific received social support were -2.20 and 1.94 , simple slopes for individuals reporting high and low levels of smoking-specific social support were significant (at the $10 \%$ level).

Similar results emerged in the second moderator analysis that predicted biochemically verified point prevalence at T2 (see Table 3, first column). Again, the interaction of volitional self-efficacy and smoking-specific received social support was significant $(p<.05$, see Figure 2$)$. The analysis of the region of significance by the Johnson Neyman technique showed that the simple slopes were significant only at levels of social support higher than $1.86(p<.05)$. In sum, the results indicate that those individuals who reported high levels of smoking-specific received social support were more likely to be abstinent, the higher their volitional self-efficacy was, indicating a synergistic effect of smoking-specific received social support and volitional selfefficacy. In contrast, individuals reporting lower levels of smoking-specific social support did not benefit from high levels of volitional self-efficacy regarding their smoking abstinence.

\section{Testing the joint effects of received social support and action and coping planning}

With the same data analytic approach as described above, we tested whether received social support moderates the association between action and coping planning and smoking cessation. Action planning did not show an interaction with received social support, neither for continuous abstinence nor for point prevalence (see Tables 2 and 3 , second column).

Coping planning showed an interaction with received social support for continuous abstinence $(p<.05$, see Table 2 , third column). The analysis of the region of significance by the Johnson Neyman technique showed that the simple slopes were only significant at levels of social support higher than $1.65(p<.05)$. As the maximum of the mean-centred smoking-specific received social support was 1.94, simple slopes were only significant for individuals reporting high levels of smoking-specific received social support. For these individuals, a very similar picture as with volitional self-efficacy emerged (see Figure 1, right panel): the combination of high coping 
planning and high social support was related to successful continuous abstinence, indicating a synergistic effect. Individuals reporting low levels of smoking-specific social support did not benefit from high levels of coping planning regarding their smoking cessation. In a second moderator analysis predicting biochemically verified point prevalence at $\mathrm{T} 2$, there was no interaction between coping planning and received social support (see Table 3, third column).

\section{Discussion}

This study aimed at testing the role of smoking-specific received social support in combination with individual self-regulation in smoking cessation. To our knowledge, this study was the first that examined the joint effects of individual and social regulation in the context of smoking cessation. Two alternative hypotheses were tested: (1) the potentially compensating function of received social support for low levels of individual regulation or (2) potential synergistic effects of individual regulation and social support.

Although we did not find direct effects of volitional self-efficacy, coping planning or smoking-specific received social support on continuous abstinence, the interaction terms of volitional self-efficacy and coping planning by smoking-specific received social support emerged as relevant predictors. Results indicate a rejection of the compensation hypothesis and rather emphasise a synergistic relationship. Individuals with high levels of received social support from their non-smoking partner are more likely to stay abstinent, the higher their volitional self-efficacy/coping planning was. Thus, the results from Warner et al. (2011), which demonstrated that for multimorbid individuals with lower self-efficacy, received social support served a compensating function with regard to perceived autonomy that could not be transferred to the context of smoking cessation. Our findings rather indicated that the stressful and taxing situation of quitting smoking requires a combination of self-regulatory and social resources, whereas the compensation of weaker self-regulatory resources (volitional self-efficacy, coping planning) by means of received social support may not be sufficient to successfully implement an intention to quit smoking. This might indeed be an effect of the difficulty of the behaviour: stopping smoking is known to be extremely difficult with relapse rates of over $70 \%$ after one month (Hughes, Keely, \& Naud, 2003). As reported above, this was quite similar in the present study. Thus, future studies should examine whether the need for high levels of received social support together with high levels of self-regulation competence is possibly less pronounced for health behaviours that are easier to implement, such as making a medical appointment.

Results for the two outcome measures of smoking cessation (self-reported continuous abstinence and biochemically verified point prevalence of abstinence) differed slightly in that the interaction effect between received support and coping planning did not gain significance for the measure of point prevalence. This measure, albeit objectively measured, is less strict than the measure of continuous abstinence. As a consequence, almost half of the people classified by this measure as non-smokers at $\mathrm{T} 2$ were by self-report not continuously abstinent. In contrast, individuals who claimed continuous abstinence were also verified by this objective point prevalence measure. Thus, the results of the continuous abstinence measure can be trusted. 
Of the three volitional constructs considered, only action planning did not interact with received social support. A potential explanation could lie in the nature of this construct and this study's design: action planning refers to specific plans about when, where and how to quit smoking. The participants in the current study set themselves a quit date which was very strict because of the study design. They showed high commitment to this date and therefore probably action planning was already obsolete. Another explanation might lie in the fact that most of the participants $(81.6 \%)$ had already tried to quit smoking prior to the study. Action planning could have been more important for individuals on their first quit attempt, as most participants in the current study were experienced in planning the cessation. As several studies demonstrated (Hughes, Keely, \& Naud, 2003), most of the smokers manage not to smoke a few days after the cessation date but then fail to maintain their smoke-free status and have relapses when difficult situations in their daily life arise. Our findings suggest that these situations can be managed with a combination of social support and coping planning, which therefore seems to be of greater importance for continuous abstinence than action planning.

Some limitations of the current study need to be addressed. Assessing smoking cessation regarding continuous abstinence as the dependent variable was self-reported. Self-reported variables might bias the validity of the assessment. However, in the context of smoking cessation, self-report is highly accurate except for clinic or other intensive intervention studies and high-risk or medical patients (Velicer, Prochaska, Rossi, \& Snow, 1992). As the study sample did not include high-risk or medical patients, self-reported continuous abstinence in the present study should be reasonably accurate. In addition, to account for potential bias, social desirability was assessed. The association between smoking cessation and social desirability was close to zero. Thus, it can be assumed that the self-reported smoking cessation is at least not biased by social desirability. Additionally, smoking status at the follow-up was biochemically verified and yielded similar results. Another limitation was the rather small effects, the findings should therefore be replicated. As the sample of the present study consisted of heterosexual smokers, who were committed to and cohabited with a non-smoking partner; generalisability of the results might be limited (e.g. regarding couples with both partners smoking and wanting to quit). Further replication studies should test different samples and also different health behaviours. Finally, the current longitudinal study included both self-regulation and received social support as perceived by the smoker. In future studies, it would be valuable to use an intensive longitudinal design including close others to get a more fine-grained picture of the effects of self-regulation and close relationships on health in daily life (Stadler, Snyder, Horn, Shrout, \& Bolger, 2012).

Despite these limitations, this study has important theoretical and practical implications. The results provide first evidence that the combination of individual and social factors is helpful for successfully quitting smoking. From a theoretical point of view, these findings argue for combining the two lines of research that have mostly been independently examined so far: models of individual health behaviour change and the role of received social support in health behaviour change (e.g. Scholz et al., 2013). Moreover, as this study demonstrates, the interplay of individual and social factors might be of even greater importance than mere main effects. From a practical perspective, interventions on smoking cessation should strengthen individual resources but also include the social environment; especially since smoking is a behaviour often performed in company and affecting the social environment. As people who live with a smoking 
partner have an increased risk for several diseases compared to those living with a non-smoker (Law, Morris, \& Wald, 1997), providing social support to help the partner to quit smoking is also beneficial for themselves.

Concluding, the study yielded the first evidence for the importance of the combination of individual and social factors in smoking cessation. Future research should explore these findings further and develop interventions to provide smokers who intend to quit with the best possible conditions.

\section{Acknowledgements}

This study and the first author were funded by the Swiss National Science Foundation (100014_124516). The second author's contribution was supported by a grant from the Ministry of Science and Higher Education (BST/WROC/2012/01). We would like to thank all students who helped with data collection.

\section{Note}

1. Participants and their partners were invited to the authors' lab in order to ensure couples completing the smoker and partner questionnaires in separate rooms. Moreover, biochemical verification of smoking status necessitated couples to come to the lab. In this study, the focus is on smokers only.

\section{References}

Ajzen, I. (1991). The theory of planned behavior. Organizational Behavior and Human Decision Processes, 50, 179 211. doi:10.1016/0749-5978(91)90020-T

Andersen, S. (2006). Do indigenous helpers foster smoking cessation in adult smokers? Addictive Behaviors, 31, 1496 1502. doi:10.1016/j.addbeh.2005.10.008

Anderson, E. S., Winett, R. A., \& Wojcik, J. R. (2007). Self-regulation, self-efficacy, outcome expectations, and social support: Social cognitive theory and nutrition behavior. Annals of Behavioral Medicine, 34, 304 312. doi:10.1007/BF02874555

Bandura, A. (2001). Social cognition theory: An agentic perspective. Annual Review of Psychology, 52, 1 26. doi:10.1146/annurev.psych.52.1.1

Burkert, S., Knoll, N., \& Scholz, U. (2005). Korrelate der Rauchgewohnheiten von Studierenden und jungen Akademikern: Das Konzept der dyadischen Planung [Correlations of smoking behavior in college students and young academics. The concept of dyadic planning]. Psychomed, 17, 240 246. doi:10.1026/0943-8149.14.3.136.

Carlson, L. E., Goodey, E., Hahn Bennett, M., Taenzer, P., \& Koopmans, J. (2002). The addition of social support to a community-based large-group behavioral smoking cessation intervention: Improved cessation rates and gender differences. Addictive Behaviors, 27, 547 559. doi: $10.1016 / \mathrm{S} 0306-4603(01) 00192-7$

Cohen, J., Cohen, P., West, S. G., \& Aiken, L. S. (2003). Applied multiple regression/correlation analysis for the behavioral sciences (3rd ed.). Mahwah, NJ: Lawrence Erlbaum Associates.

Fisher, J. D., Fisher, W. A., Amico, K., \& Harman, J. J. (2006). An information-motivationbehavioral skills model of adherence to antiretroviral therapy. Health Psychology, 25, 462 473. doi: $10.1037 / 0278-6133.25 .4 .462$

Graham, J. W. (2009). Missing data analysis: Making it work in the real world. Annual Review of Psychology, 60, 549 576. doi:10.1146/anmurev.psych.58.110405.085530 
Gulliver, S. B., Hughes, J. R., Solomon, L. J., \& Dey, A. N. (1995). An investigation of self-efficacy, partner support and daily stresses as predictors of relapse to smoking in self-quitters. Addiction, 90, 767 772. doi: $10.1046 /$ j.1360-0443.1995.9067673.x

Gwaltney, J. M., Metrik, J., Kahler, C. W., \& Shiffman, S. (2009). Self-efficacy and smoking cessation: A meta-analysis. Psychology of Addictive Behaviors, 23, 56 66. doi:10.1037/ a0013529

Hamilton, K., \& White, K. M. (2008). Extending the theory of planned behavior: The role of self and social influences in predicting adolescent regular moderate-to-vigorous physical activity. Journal of Sport \& Exercise Psychology, 30, 5674.

Hughes, J. R., Keely, J., \& Naud, S. (2003). Shape of the relapse curve and long-term abstinence among untreated smokers. Addiction, 99, 29 38. doi:10.1111/j.1360-0443.2004.00540.x

Hughes, J. R., Keely, J. P., Niaura, R. S., Ossip-Klein, D. J., Richmond, R. L., \& Swan, G. E. (2003). Measures of abstience in clinical trials: Issues and recommendations. Nicotine \& Tobacco Research, 5, 13 25. doi:10.1080/1462220031000070552

Keller, R., Radtke, T., Krebs, H., \& Hornung, R. (2011). Der Tabakkonsum der Schweizer Wohnbevölkerung in den Jahren 2001 bis 2010. Tabakmonitoring Schweizerische Umfrage zum Tabakkonsum [Tobacco consumption of the Swiss population between 20012011. Tobacco monitoring Switzerland Swiss survey of tobacco consumption]. Zurich: Department of Psychology, Social and Health Psychology, University of Zurich.

Law, M. R., Morris, J. K., \& Wald, N. J. (1997). Environmental tobacco smoke exposure and ischaemic heart disease: An evaluation of the evidence. British Medical Joumal, 315, 973 980. doi: $10.1136 / \mathrm{bmj} .315 .7114 .973$

McClelland, G. H., \& Judd, C. M. (1993). Statistical difficulties of detecting interactions and moderator effects. Psychological Bulletin, 114, 376 390. doi:10.1037/0033-2909.114.2.376

McMahon, S. D., \& Jason, L. A. (1998). Stress and coping in smoking cessation: A longitudinal examination. Anxiety, Stress \& Coping: An International Journal, 11, 327 343. doi:10.1080/ 10615809808248318

Mokdad, A. H., Marks, J. S., Stroup, D. F., \& Gerberding, J. L. (2004). Actual causes of death in the united states, 2000. JAMA, 291, 1238 1245. doi:10.1001/jama.291.10.1238

Park, E. W., Tudiver, F., Schultz, J. K., \& Campbell, T. (2004). Does enhancing partner support and interaction improve smoking cessation? A meta-analysis. Annals of Family Medicine, 2, 170 174. doi: $10.1370 / \mathrm{afm} .64$

Povey, R., Conner, M., Sparks, P., Rhiannon, J., \& Shepherd, R. (2000). The theory of planned behaviour and healthy eating: Examining additive and moderting effects of social influence variables. Psychology \& Health, 14, 991 1006. doi:10.1080/08870440008407363

Preacher, K. J., Curran, P. J., \& Bauer, D. J. (2006). Computational tools for probing interaction effects in multiple linear regression, multilevel modeling, and latent curve analysis. Journal of Educational and Behavioral Statistics, 31, 437 448. doi:10.3102/10769986031004437

Prochaska, J. O., \& DiClemente, C. C. (1983). Stages and processes of self-change of smoking: Towards an integrative model of change. Journal of Consulting and Clinical Psychology, 51, 390 395. doi: $10.1037 / 0022-006 X .51 .3 .390$

Sarason, I. G., Sarason, B. R., \& Shearin, E. N. (1986). Social support as an individual difference variable: Its stability, origins, and relational aspects. Journal of Personality and Social Psychology, 50, 845 855. doi:10.1037/0022-3514.50.4.845

Scholz, U., Nagy, G., Göhner, W., Luszczynska, A., \& Kliegel, M. (2009). Changes in selfregulatory cognitions as predictors of changes in smoking and nutrition behaviour. Psychology \& Health, 24, 545 561. doi:10.1080/08870440801902519

Scholz, U., Ochsner, S., Hornung, R., \& Knoll, N. (2013). Does social support really help to eat a low-fat diet? Main effects and gender differences of received social support within the Health Action Process Approach. Applied Psychology: Health and Well-Being, 5, 270 290. doi:10.1111/ aphw. 12010 
Schwarzer, R. (2008). Modeling health behavior change: How to predict and modify the adoption and maintenance of health behaviors. Applied Psychology: An International Review, 57, 129. doi:10.1111/j.1464-0597.2007.00325.x

Schwarzer, R., \& Gutierrez-Dona, B. (2005). More spousal support for men than for women: A camparison of sources and types of support. Sex Roles, 52, 523 532. doi:10.1007/s11199005-3718-6

Schwarzer, R., \& Knoll, N. (2010). Social support. In J. W. A. Kaptein \& J. Weinman (Eds.), Health Psychology (pp. 283 293). Oxford: Blackwell.

Schwarzer, R., \& Luszczynska, A. (2008). How to overcome health-compromising behaviors: The health action process approach. European Psychologist, 13, 141 151. doi:10.1027/10169040.13.2.141

Sillars, A. L., \& Scott, M. D. (1983). Interpersonal perception between intimates. An integrative review. Human Communication Research, 10, 153 176. doi:10.1111/j.1468-2958.1983. tb00009.x

Sniehotta, F. F., Schwarzer, R., Scholz, U., \& Schuez, B. (2005). Action planning and coping planning for long-term lifestyle change: Theory and assessment. European Journal of Social Psychology, 35, 565 576. doi:10.1002/ejsp. 258

Stadler, G., Snyder, K. A., Horn, A. B., Shrout, P. E., \& Bolger, N. (2012). Close relationships and health in daily life: A review and empirical data on intimacy and somatic symptoms. Psychosomatic Medicine, 74, 398 409. doi:10.1097/PSY.0b013e31825473b8

Stöber, J., \& Luther, M. (2001). The social desirability scale-17 (SDS-17). Convergent validity, discriminant validity, and relationship with age. European Journal of Psychological Assessment, 17, 222 232. doi:10.1027//1015-5759.17.3.222

Tabachnick, B. G., \& Fidell, L. S. (2001). Using multivariate statistics (4th ed.). Boston, MA: Allyn and Bacon.

Velicer, W. F., Prochaska, J. O., Rossi, J. S., \& Snow, M. G. (1992). Assessing outcome in smoking cessation studies. Psychological Bulletin, 111, 23 41. doi:10.1037/0033-2909.111.1.23

Warner, L. M., Ziegelmann, J. P., Schuez, B., Wurm, S., Tesch-Roemer, C., \& Schwarzer, R. (2011). Maintaining autonomy despite multimorbidity: Self-efficacy and the two faces of social support. European Journal of Ageing, 8, 3 12. doi:10.1007//s10433-011-0176-6

West, R., Hajek, P., Stead, L., \& Stapleton, J. (2005). Outcome criteria in smoking cessation trials: proposal for a common standard. Addiciton, 100, 299 303. doi:10.1111/j.13600443.2005.0095. $\mathrm{x}$

World Health Organization (WHO). (1998). Guidelines for controlling and monitoring the tobacco epidemic. Geneva: World Health Organization. 